\title{
A Reconfigurable Multi-standard Radio Platform
}

\author{
Antti Immonen, Aarno Pärssinen, Tommi Zetterman \\ Nokia Corporation, Nokia Research Center \\ P.O. Box 407, FI-00045 Nokia Group, Finland \\ \{antti.immonen, aarno.parssinen, tommi.zetterman\}@nokia.com \\ Mikko Talonen, Jussi Ryynänen, Sami Kiminki, Vesa Hirvisalo \\ Aalto University \\ P.O. BOX 13000, FI-00076 Aalto, Finland \\ \{mikko.talonen, jussi.h.ryynanen, \\ sami.kiminki, vesa.hirvisalo\}@tkk.fi
}

\begin{abstract}
This paper presents a dynamically reconfigurable multi-radio RF architecture concept, which can be used for RF platform and control optimization. The platform realization is based on the RF hardware and its configuration mechanisms. The related control software is realized through functional separation of configuration management and timing control. Both key hardware and software elements are discussed and optimization opportunities evaluated using high-level analysis on key building blocks.
\end{abstract}

Keywords. RF architecture, SDR, Multiradio, dynamic scheduling

\section{Introduction}

The complexity of the mobile radio communication platforms is rising rapidly. At the same time the mobile industry is under consolidation and pressure to reduce investments. These two issues together pose new kind of challenges for the ecosystem.

Modern multimedia phones and mobile internet devices (MIDS) are already having multiple radio protocols to support different tasks and services they provide for consumers.

In current approach, the communication systems are mostly implemented by using dedicated hardware (HW) for each communication protocol. It is not easy to provide flexibility to optimize the resources and to minimize energy consumption when multiple radio protocols are operated using the same HW. This is aggravated when the dynamic behavior of different protocols is taken into account. In order to be capable of managing and optimizing the platform during the design phase and especially during the run-time, one must develop a 


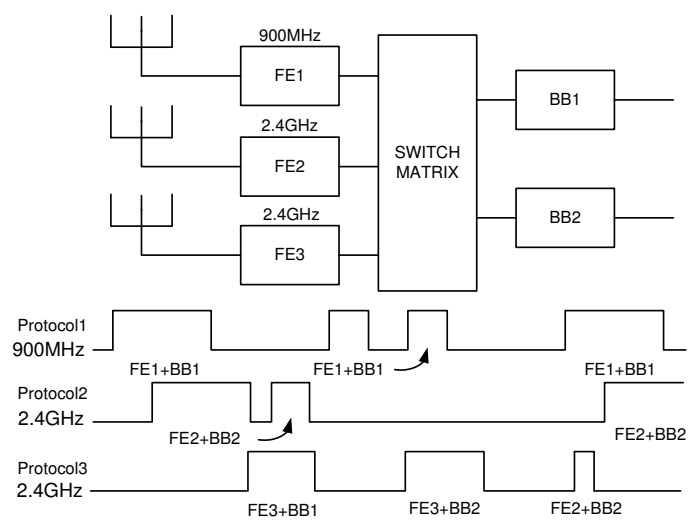

Figure 1: Concept of configurable RF

rigorous framework and abstraction scheme for the control of the multi-radio RF. The purpose of the abstraction is to avoid too sturdy and cumbersome control schemes. Furthermore, abstraction allows platform-independent software (SW) development without detailed information of underlying HW. In a radio, analog $\mathrm{RF}$ processing is a specific entity because it cannot utilize any memory during transmission and reception. Hence, scheduling must be based on the radio protocols occupying complete but programmable and flexible RF signal chains. In a software-defined radio (SDR) RF platform, a part of the HW components could be made tunable to serve multiple protocols.

The problem of co-existence of radios has been addressed for example in [1] and a solution for a MAC based on functionality splitting has been proposed [2]. We address the same problem domain, but using protocol-assisted isolation mechanisms that leave space for various solutions of radio cognition [3].

The selected approach creates a usage-centric platform control framework such that the RF and its control are abstracted from the protocol layers, i.e., the platform is transport-independent. In this context, transport-independence means that none of the protocols have dedicated resources for their requests.

The resources needed to perform the tasks requested by the applications (i.e., the radio protocols) are solely allocated and controlled locally by the control framework.

This paper is organized as follows. Section 2 presents the system architecture. The proposed RF platform and RF resource management are presented in Sections 3 and 4. Section 5 presents an analysis on hardware for various platform configurations.

\section{System Architecture}

The key challenge for the system architecture [4] is to have flexibility to assign different radio systems independently onto an SDR. In baseband processing this can rely on vector processing or other computing elements. The concept of configurable RF is presented in Figure 1. The HW has multiple, configurable signal chains both for transmission and reception. The emphasis in this paper is on the receiver-side. However, the same approach applies to transmitter-side 


\begin{tabular}{|c|}
\hline Radio protocols \\
\hline RF Services \\
$>$ Radio system \& mode, RF band \& BB bandwidth \\
$>$ RF performance \& reports \\
\hline RF Configuration \\
$>$ ASIC functionality vs. FE functionality \\
\hline RF Performance \\
$>$ Performance adjustment: global (Power ctrl), local (calibration) \\
\hline Mapping to implementation \\
$>$ control of IP blocks
\end{tabular}

Figure 2: Abstracted RF control

as well. The desired configurations can be made by multiplexing the switch matrix. This is a good approximation of the configuration opportunities that state-of-the-art RF transceivers can provide. RF blocks are assumed to be flexible enough to support multiple protocols with known band limitations. For example, block BB1 is used by protocols 1 and 3 in this figure.

In the foreseeable demanding use cases, two or more protocols out of 5-10 protocols could be active at a time. Most of the activated protocols are merely maintaining links to guarantee mobility. Hence, it is reasonable to assume that the resource-hungry high-speed traffic is carried out by one or at most by two of the protocols at a time. To allow the link-maintaining and the low-speedtraffic radios to share a signal chain, the RF subsystem must support dynamic scheduling and configuration of the hardware.

$\mathrm{RF}$ band and performance requirements will have major impact on the configuration. Not all of the RF elements can be stretched to support all frequency bands and operating conditions. Thus, the performance cannot be controlled without HW-specific information. However, performance information does not need to be protocol-specific by nature.

Radio standards specify requirements for performance, but do not define the implementation. For an RF platform, the key differences between protocols that need to be taken into account are radio frequency bands, channel bandwidths, sensitivity (i.e., noise performance) and selectivity (i.e., ability to tolerate interference). Due to dynamic nature and a complex set of different requirements over different protocols, the performance tuning should have an abstracted overlay with other configuration parameters to be manageable on a multi-radio platform. An approach towards abstracted RF control is described in Figure 2.

Only some of the protocol specifications guarantee interoperability with the others. For example, internal interoperability between GSM, EDGE, WCDMA, HSPA, and LTE guarantees that those can use the same resources to some extent and perform necessary monitoring actions without multiplying the HW. Thus, because of the variance and the number of the protocols involved in contemporary smart phones, the analysis of the requirements is exhaustive. To cope with the future complexity, mechanisms at a suitable abstraction level are needed to ensure realization of interoperability on a multi-radio platform.

Our system architecture consists of a reconfigurable RF platform, platform control, digital baseband processing (PHY \& MAC) and network stacks, as illustrated in Figure 3. The platform control consists of a multi-radio controller, 


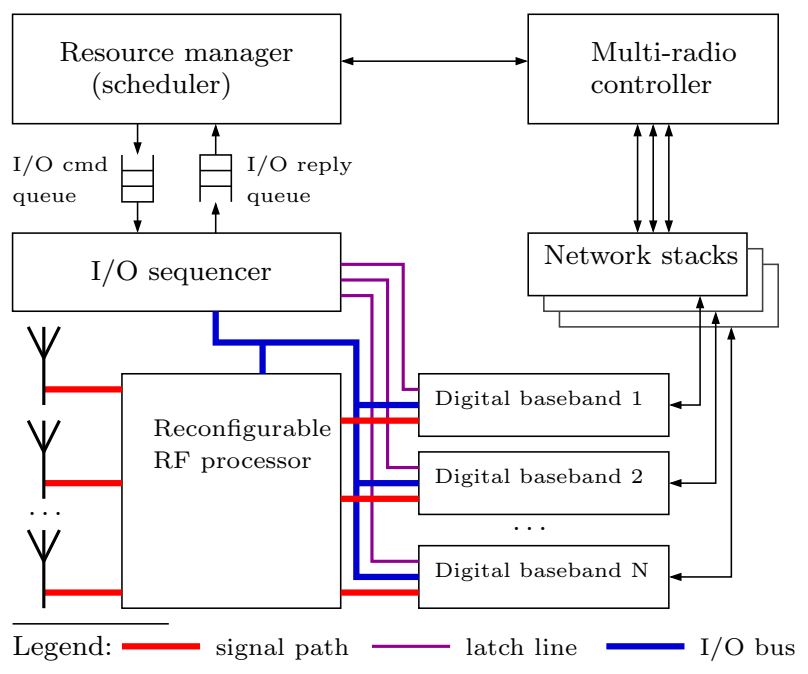

Figure 3: The overall system architecture

a resource manager and an $\mathrm{I} / \mathrm{O}$ sequencer. The multi-radio controller can synchronize transmissions of mutually interfering radio protocols, such as $802.11 \mathrm{~g}$ and Bluetooth. The resource manager maintains schedules for the configuration of the RF platform. The schedules are executed by the I/O sequencer to achieve precise timing in $\mathrm{RF}$ configuration and transmission latching.

$\mathrm{HW}$ resource scheduling is driven by the protocols and the multi-radio controller. This enables scheduling of specific HW resources, as resource demand can be estimated in advance.

Flexibility and predictability are important scheduling characteristics and they vary between protocols.

To support sub-microsecond precision for platform reconfiguration, we use hardware-assistance in configuring and scheduling the use of HW resources.

\section{RF Platform}

In conventional implementations, it is not possible to do any run-time reconfiguration or run-time resource sharing between protocols. To illustrate the situation, we show an example of a possible implementation of a GSM/WCDMA/ LTE/WLAN receiver in Figure 4. The solution reflects a current state-of-the-art WEDGE receiver [5] with added LTE functionality at the 2.5-GHz band. LTE can be adopted at various cellular bands but here we have limited the cellular MIMO functionality, required in LTE, only to one band. This does not restrict the approach being used in more complex scenarios.

The key technology limitations are related to RF filters. Current technology does not enable implementations of tunable RF filters with adequate performance. This poses a major obstacle especially in near future when the number of systems and frequency bands is increasing.

Within the industry, substantial amount of work has been done to achieve more flexibility in HW separately in cellular and WLAN domains [5-8]. Despite of more flexible HW architectures, increased number of specified frequency 


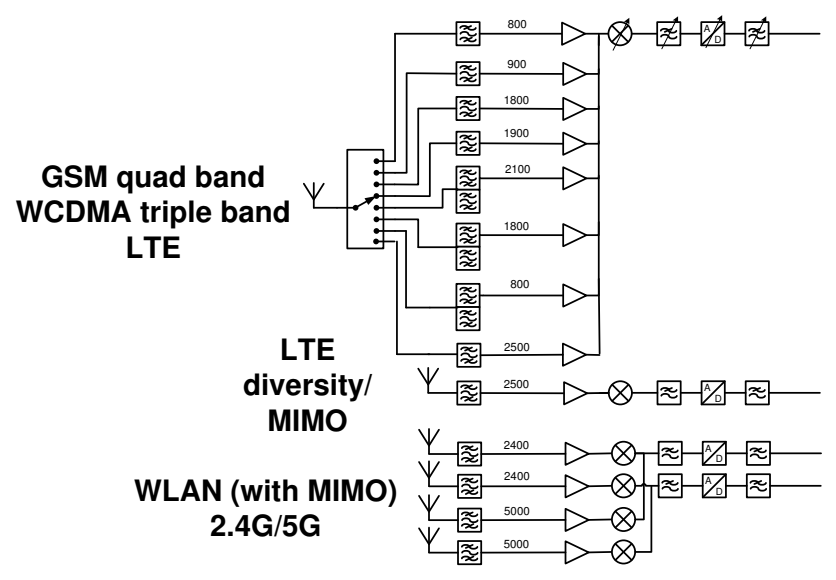

Figure 4: Receiver architecture with dedicated resources

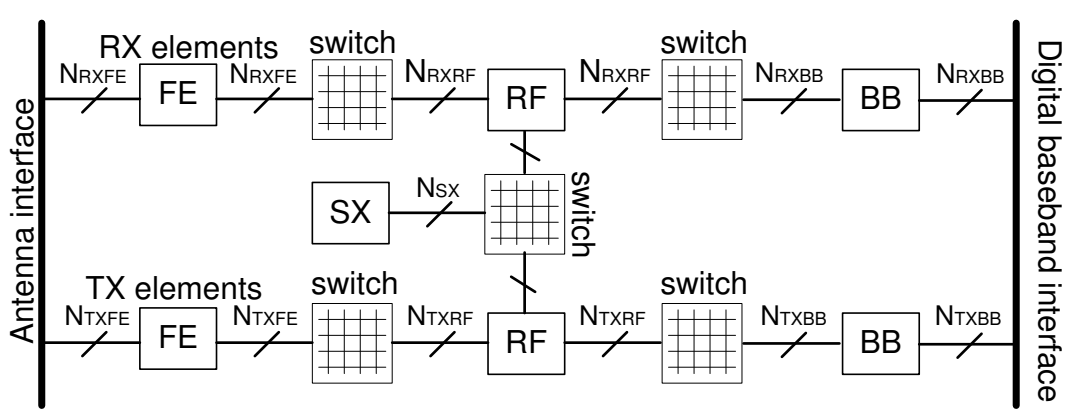

Figure 5: Logical separation of RF elements

bands combined with limited tunability of RF filters is shifting the complexity to even more parallel radio frequency processing. Emerging MIMO technology will further complicate this issue in adding more parallel signal paths to the HW platform. Cellular communications is an excellent example of resource sharing opportunity embedded into protocols where second and third generation systems (GSM/EDGE \& WCDMA/HSPA) could share most of the low-frequency processing elements and the same is foreseeable in the fourth generation (LTE). In addition, solutions where systems share resources with customized interoperability mechanisms have been presented. For example, GPS has been adapted to diversity path of an RF solution [9]. Dynamic resource sharing and run-time reconfigurability can be enhanced to allow concurrent operation of multiple protocols using at least some shared resources in the platform. However, this requires improved scheduling awareness and careful characterization of realistic use scenarios as described later in this paper.

The RF platform model in the analysis consists of parallel processing elements that can be interconnected flexibly to form desired transmitter (TX) and receiver (RX) chains. Processing blocks are divided in different logical categories based on physical constraints, interconnection possibilities, and functionality of the blocks. The following logical entities, illustrated in Figure 5, can be identified: RF front-end (RF FE), RF ASIC, analog baseband (BB), and synthesizer 
(SX). This division is natural, and reflects the functionalities of the blocks.

In this scheme, RF FE consists of antennas, switches, and RF filters both in RX and TX. RF FE-ASIC interface is the first natural boundary from antenna in design space. Hence any external TX power amplifiers (PA's) and RX low noise amplifiers (LNA's) should be included into RF FE. These RF FE blocks can be interconnected to a set of RF ASIC elements that carry out other RF processing functionalities (amplification, frequency conversion, etc.) and again the RF ASIC blocks can be connected to BB processing entities. Synthesizer (SX) is solely responsible for the generation of LO frequencies for RX and TX chains.

The performance of the logical blocks has to be described sufficiently for the analysis. However, the level of modeling should be kept as simple as possible to avoid excessive complexity. RF frequency analysis is on the top of the analysis. For that reason, RF frequency range and $\mathrm{BB}$ bandwidth are the key parameters. In synthesizer, frequency range and frequency step are the most important parameters. Anyhow, the level of modeling is not restricted to these and conventional performance analysis is one of the underlying elements as shown in Figure 2.

Configuration of RX and TX chains for a specific protocol is based mainly on frequency range characteristics of processing elements. Other underlying key parameters such as noise and linearity can also be used, if the configuration scheme is built such that it is capable to take into account the clear dependence with these parameters and the RF frequency and bandwidth.

\section{RF Resource Management}

Our management of HW resources is based on separation of tasks between the resource manager, multi-radio controller and I/O sequencer. The concept is general and designed especially for the management of shared hardware blocks such as RF signal processing chains and hardware accelerators.

The resource manager ties radio protocol drivers to the reconfigurable multiradio platform by mapping $\mathrm{RF}$ requirements to platform configurations. Thus, protocol drivers are isolated from the hardware by hiding the actual configuration used in task execution.

The multi-radio controller does the resource allocation, the configuration management, the scheduling, and the policy management needed in managing the RF HW. It also detects and solves spectral conflicts between radios.

An active radio is in one of several radio-specific operational states characterized by its communication behavior and resource usage. Operational states capture the varying resource demands when the radio is performing different kinds of activity (e.g., communicating, scanning, maintaining link, etc). Resource allocation is based on characteristics of radio usage, such as frequency band and RF resource utilization properties. These are used to dynamically determine whether the platform can serve the request. In case of insufficient resources, the request cannot be granted. On the other hand, extra resources can be used, e.g., to enable MIMO for some radio.

The configuration management finds alternative configurations of real hardware for realizing radio resource requests. It assigns a cost parameter to each alternative configuration based on performance and energy consumption esti- 


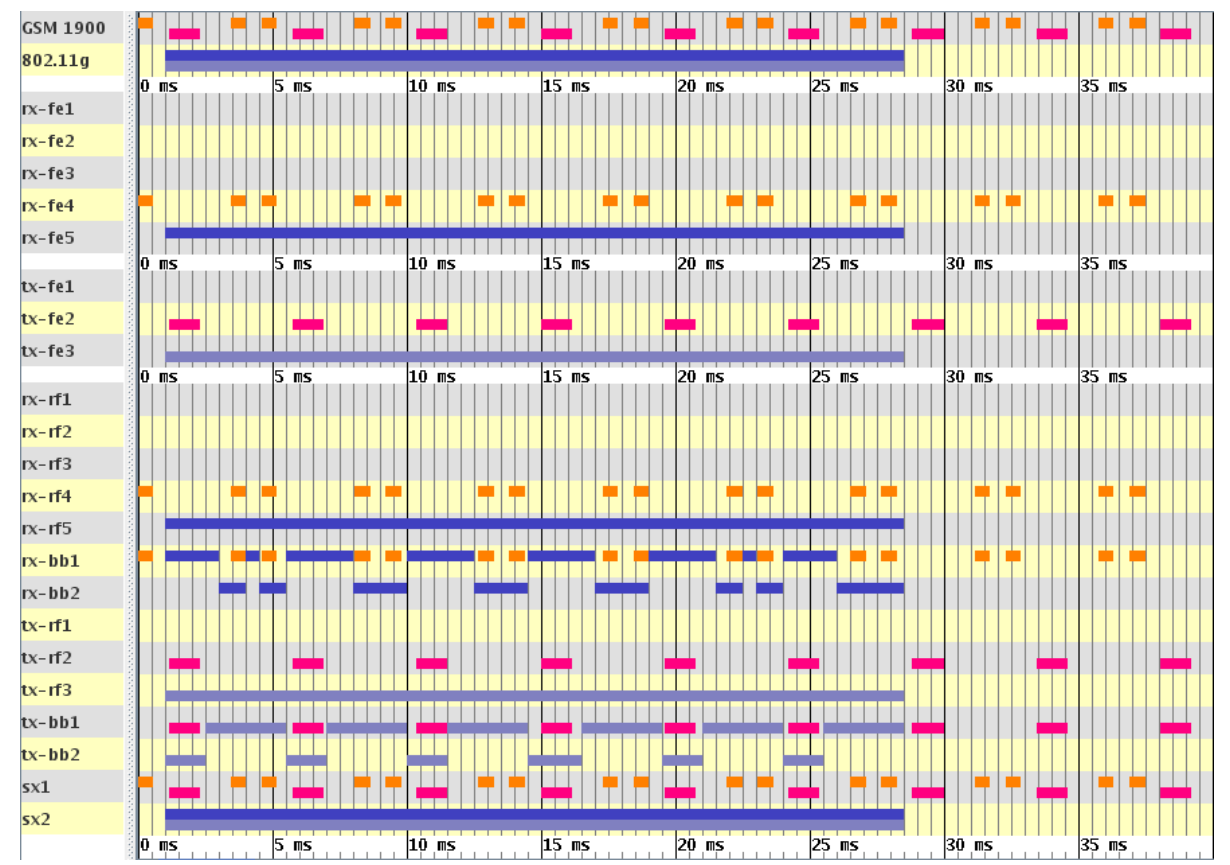

Figure 6: Example of allocation scheduling. In each row, the upper half denotes allocation for RX tasks and the lower half is for TX tasks, respectively.

mates and interference. This enables optimizations in resource scheduling, such as choosing cheaper-to-use resources in good transmission conditions.

The reconfiguration scheduler processes RF utilization requests from protocol drivers and, in conjunction with the configuration manager, produces scheduling of hardware resources. The allocation schedules for individual HW resources are maintained proactively for the future in the extent that the protocol drivers can predict their workload.

Schedules are maintained by software and their execution is done by sequencing hardware. Considering the software, better than $1 \mathrm{~ms}$ reconfiguration response times should be obtainable for new utilization requests. For protocols with sufficient predictability, each RF resource requirement can be processed individually. These include current 3GPP protocols, even in the case of LTE HARQ processing. However, some protocols, e.g., the 802.11 family, require considerably tighter response times. In these cases, instead of allocating RX/TX jobs, potential for RX/TX job execution is allocated.

An example simulation run is presented in Figure 6, which handles a combination of GSM 1900 and WLAN 802.11g load by using switch topology of Figure 5. In RX, front-end (rx-feX) and RF (rx-rfX) elements 1-4 are dedicated for the four GSM band, and elements (rx-fe5, rx-rf5) are for WLAN. Baseband elements (rx-bbX) are symmetric. In TX, tx-fe1 and tx-rf1 are used for GSM $850 / 900$ bands, tx-fe2 and tx-rf2 are used for GSM 1800/1900 bands, and tx-fe 3 and tx-rf3 are used for WLAN. Baseband elements (tx-bbX) are symmetric also in TX. Signal generators are shared between RX and TX tasks.

As shared resources are utilized, resource conflicts are sometimes unavoid- 


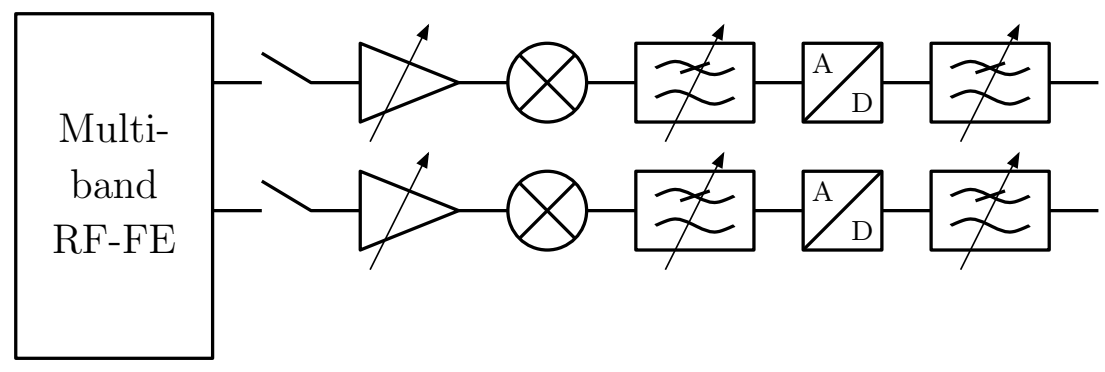

Figure 7: Flexible receiver

able. This could be due to mutually interfering radio tasks or simply because of momentarily insufficient available resources. Fortunately, RF protocols are designed to tolerate some level of skipped or lost TX and RX transmissions, and therefore, conflicts are acceptable as long as the skip probability is low enough (e.g., < 1\%). When conflicts do happen, the priority assigned by the policy manager is used to determine which jobs are skipped.

Finally, as the utilization load is predictable to at least few milliseconds in future, it is possible to almost always find predictable idle slots in resource schedules. These can be used to execute background RF tasks - such as spectrum sensing - and background radio protocols. We expect this functionality to be useful in the next generation cognitive radio protocols.

\section{Platform Configuration HW Analysis}

Having flexible RF platform enabling run-time reconfiguration and resource sharing it is possible to reduce the number of HW components. Energy consumption does not automatically scale down with the number of required components, but reductions are also possible as described later in this section. In general, the possibility to utilize same resources depends on the time domain behavior of the protocols, the ability to schedule them, and their frequency bands. For example, LTE band 7 and WLAN could, in principle, share HW resources because they both utilize packet-form transmission and reception and the operating frequencies are close enough (WLAN $2.4 \mathrm{GHz}$ and LTE $2.5 \mathrm{GHz}$ ). However, this may lead to significant data rate reductions if the use scenarios are not carefully considered. Here we assume that only one protocol (cellular or WLAN) will transfer information at peak data rate and the other's link can be fully functional but both will not provide full data rate connection simultaneously.

One of the essential issues in HW platform design is to minimize the area and energy consumption in order to save costs. Where Moore's law ensures the area reduction of digital processing, $\mathrm{RF} \mathrm{HW}$ does not scale accordingly. This calls for other means to reduce cost. Cost is mostly linked to the area and thus to the actual numbers of HW block used in the implementation. In addition to this, the energy consumption of the implementation can be regarded as cost. To show the benefits of a flexible reconfigurable RF platform, we analyze different receiver implementation approaches with respect to configurability.

The system portfolio is the following in our analysis: quad-band GSM, triple- 
band WCDMA, LTE with 2x MIMO capabilities + WLAN $2.4 / 5 \mathrm{GHz}$ with $2 \mathrm{x}$ MIMO capabilities. This kind of feature set depicts realistically the required functionality of future receivers.

One can see three HW approaches to construct an RF receiver:

1. Fully system-dedicated receiver chain where protocols use only resources dedicated for them

2. More tunable but still frequency selective receiver chain

3. Fully broadband receiver chain

In approach 1, resources are dedicated for the used protocols and thus there is no flexibility. In approach 2 , the blocks can be tuned as follows. RF can be tuned between frequency ranges of $0.4-1.0 \mathrm{GHz}, 0.8-2 \mathrm{GHz}, 1.7-2.6 \mathrm{GHz}$, and 2.4-5.5 GHz. BB can be tuned to handle all required bandwidths. In approach 3 , it is assumed that RF and BB can be tuned to cover the whole frequency range. In all of these approaches, the SX frequency range is assumed to be 0.5-5.5 GHz. However, an SX may consist of more than one VCO cores due to restricted frequency tuning range. Cellular requires one SX for RX and another for TX in FDD modes used in WCDMA and LTE. WLAN requires one SX, which is shared by RX and TX.

The receiver presented in Figure 4 depicts approach 1 . The corresponding most flexible implementation utilizing commercially presented technology is presented in Figure 7. In Figure 7, the multi-band RF-FE is similar as in Figure 4.

We discuss four different classes of worst-case use cases:

A. Cellular $2 \mathrm{G} / 3 \mathrm{G} / 4 \mathrm{G}$ (LTE only at $2.5 \mathrm{GHz}$ with MIMO) + WLAN 802.11n (MIMO)

B. Cellular $2 \mathrm{G} / 3 \mathrm{G} / 4 \mathrm{G}$ (LTE only at $2.5 \mathrm{GHz}$ with MIMO) + WLAN $802.11 \mathrm{a} / \mathrm{g}(\mathrm{SISO})$

C. Cellular SISO (2G/3G) + WLAN 802.11n (MIMO)

D. Cellular $2 \mathrm{G} / 3 \mathrm{G} / 4 \mathrm{G}$ (LTE only at $2.5 \mathrm{GHz}$ with MIMO) prioritized, WLAN $(802.11 \mathrm{a} / \mathrm{g} / \mathrm{n})$ can send/receive packets only if cellular is not allocating traffic (run-time reconfiguration is possible if platform construction allows it)

Based on simulations and analysis, the number of HW entities needed to fulfill the required functionality in these approaches is collected in Table 1 . The conventional approach 1 results in the highest number of HW entities. Due to dedicated resources, especially the number of $\mathrm{RF}$ entities is significantly larger than in other approaches. Approaches 2 and 3 differ only in the numbers of RF entities. Anyhow, approach 3 cannot by default be claimed to be better among these two due to the different kind of characteristics of the approaches. In general, energy consumption is naturally related to the implementation techniques and especially to the bandwidth, the more wideband implementation the more energy it consumes. The fully broadband receiver in approach 3 consumes more power than the receiver in approach 2 and is more vulnerable to interferences due to the wideband nature. Thus, the design phase platform optimization is vital when considering trade-offs such as power consumption versus entity count or tolerance to interference versus number of RF entities. The lowest numbers of HW RF and HW BB entities are reached in use cases where run-time resource sharing is utilized. These cases are highlighted in Table 1. In platform design phase, the block-level energy consumption and interference issues should also be 
Table 1: Number of HW entities with different approaches and use cases

\begin{tabular}{|r|c|c|c|}
\hline Approach/Use case & RF & BB & SX \\
\hline $1 / \mathrm{A}$ & 13 & 4 & 2 \\
\hline $1 / \mathrm{B}$ & 10 & 3 & 2 \\
\hline $1 / \mathrm{C}$ & 12 & 3 & 2 \\
\hline $1 / \mathrm{D}$ & $\mathbf{1 3}$ & $\mathbf{4}$ & $\mathbf{2}$ \\
\hline $2 / \mathrm{A}$ & 5 & 4 & 2 \\
\hline $2 / \mathrm{B}$ & 4 & 3 & 2 \\
\hline $2 / \mathrm{C}$ & 4 & 3 & 2 \\
\hline $2 / \mathrm{D}$ & $\mathbf{3}$ & $\mathbf{2}$ & $\mathbf{2}$ \\
\hline $3 / \mathrm{A}$ & 4 & 4 & 2 \\
\hline $3 / \mathrm{B}$ & 3 & 3 & 2 \\
\hline $3 / \mathrm{C}$ & 3 & 3 & 2 \\
\hline $3 / \mathrm{D}$ & $\mathbf{2}$ & $\mathbf{2}$ & $\mathbf{2}$ \\
\hline
\end{tabular}

considered in addition to HW entity counts. In platform usage, the block-level energy consumption and interference are the basis for cost functions on which the most optimal resource allocations are based as described in Section 4.

\section{Conclusions}

In this paper a run-time reconfigurable platform was presented. The approach introduces abstraction concept of the platform that is required to utilize the presented scheduling and run-time reconfiguration schemes.

The run-time RF resource management concept combined with run-time reconfigurable RF platform offers chances to decrease the number of $\mathrm{HW}$ entities and also in certain conditions to lower the energy consumption. It offers possibilities to do design-time platform exploration where trade-offs such as the number of HW entities versus power consumption can be considered. Most importantly, it enables run-time platform optimization where the most energy efficient platform resource combinations can be allocated for each use scenario.

The presented approach offers new opportunities to bring SDR features more effectively into multi-radio solutions. Physical bottlenecks related to certain RF components, such as filters, cannot be overcome easily. However, efficient scheduling and possibility to protocol-independent platform control can allow enhanced complexity and adopt rapidly new improved technologies to minimize cost and power consumption.

\section{References}

[1] J. Zhu, A. Waltho, X. Yang, and X. Guo, "Multi-radio coexistence: Challenges and opportunities," in Proceedings of the 16th International Conference on Computer Communications and Networks (ICCCN), Aug. 2007, pp. 358-364.

[2] G. Nychis, T. Hottelier, Z. Yang, S. Seshan, and P. Steenkiste, "Enabling MAC protocol implementations on software-defined radios," in Proceedings of the 6th USENIX Symposium on Networked Systems Design and Implementation (NSDI), 2009, pp. 91-105. 
[3] S. Geirhofer, L. Tong, and B. M. Sadler, "A cognitive framework for improving coexistence among heterogeneous wireless networks," in IEEE Global Telecommunications Conference (GLOBECOM), Dec. 2008, pp. 1-5.

[4] A. Ahtiainen, K. van Berkel, D. van Kampen, O. Moreira, A. Piipponen, and T. Zetterman, "Multi-radio scheduling and resource sharing on a software defined radio computing platform," in Proceedings of the SDR Forum, 2008.

[5] O. Gaborieau et al., "A SAW-less multiband WEDGE receiver," in IEEE International Solid-State Circuits Conference-Digest of Technical Papers (ISSCC), Feb. 2009, pp. 114-115.

[6] A. Hadjichristos et al., "Single-chip RF CMOS UMTS/EGSM transceiver with integrated receive diversity and GPS," in IEEE International Solid-State Circuits ConferenceDigest of Technical Papers (ISSCC), Feb. 2009, pp. 118-119,119a.

[7] M. Zargari et al., "A dual-band CMOS MIMO radio SoC for IEEE 802.11n wireless LAN," IEEE Journal of Solid-State Circuits, vol. 43, no. 12, pp. 2882-2895, Dec. 2008.

[8] V. Giannini et al., "A $2 \mathrm{~mm}^{2} 0.1$-to-5GHz SDR receiver in 45nm digital CMOS," in IEEE International Solid-State Circuits Conference-Digest of Technical Papers (ISSCC), Feb. 2009, pp. 408-409.

[9] V. Aparin et al., "A fully-integrated highly linear zero-IF CMOS cellular CDMA receiver," in IEEE International Solid-State Circuits Conference-Digest of Technical Papers (ISSCC), Feb. 2005, pp. 324-325,601. 